\title{
Outage Analysis of Partial Relay Selection Schemes with Feedback Delay and Channel Estimation Errors in Nonidentical Rayleigh Fading Channels
}

\author{
Jung-Bin Kim' and In-Ho Lee ${ }^{2}$ \\ ${ }^{1}$ Broadcasting \& Telecommunications Media Research Laboratory, Electronics \& Telecommunications Research Institute, \\ Daejeon 305-700, Republic of Korea \\ ${ }^{2}$ Department of Electrical, Electronic and Control Engineering, Hankyong National University, Anseong 456-749, Republic of Korea \\ Correspondence should be addressed to In-Ho Lee; ihlee@hknu.ac.kr
}

Received 29 April 2016; Accepted 10 November 2016; Published 3 January 2017

Academic Editor: Larbi Talbi

Copyright (C) 2017 J.-B. Kim and I.-H. Lee. This is an open access article distributed under the Creative Commons Attribution License, which permits unrestricted use, distribution, and reproduction in any medium, provided the original work is properly cited.

\begin{abstract}
This paper investigates the impact of the channel estimation error and outdated channel state information (CSI) on the outage performances of partial relay selection (PRS) and efficient partial relay selection (EPRS). Considering imperfect channel estimation and outdated CSI with decode-and-forward (DF) relaying strategy, closed-form expressions for exact outage probabilities and asymptotic outage probabilities for PRS and EPRS are provided assuming independent and nonidentically distributed Rayleigh fading channels. Numerical investigations verify the analytical expression for outage probability and show how much performance is degraded by the channel estimation errors and the feedback delay that causes the outdated CSI.
\end{abstract}

\section{Introduction}

Cooperative relaying has attracted great attention because it offers an excellent performance at low cost by forming a virtual multiple-input multiple-output (MIMO) system using all available nodes as relays. In industry, the cooperative relaying system has been included in both worldwide interoperability for microwave access (WiMAX) [1] and long-term evolution (LTE) [2] to extend the cell coverage and improve the cell-edge user throughput. Among various cooperative diversity techniques, the best relay selection (BRS) is one of the most promising schemes since it can achieve full spatial diversity from multiple relays with low complexity [3], even in the presence of interference [4]. To further reduce overhead and complexity of BRS, partial relay selection (PRS) was introduced in [5], in which a single relay is selected based on only the first-hop channel state information (CSI). Hence, PRS can prolong lifetime of energy-constrained relay node. Despite such advantages, achievable performance of PRS is severely bounded because partial CSI cannot sufficiently represent the end-to-end channel quality [6]. To improve the performance of PRS with a small additional overhead, efficient partial relay selection (EPRS) was introduced in [7]. In EPRS, a link with the smaller average channel power between the first and the second hops is chosen at each endto-end path, and the CSI for the links chosen at every endto-end path is used for a single relay selection. Since the CSI used for EPRS is more correlated with the end-to-end channel quality than for PRS, EPRS can attain better performance than PRS $[7,8]$.

The assumption of perfect CSI for selecting a single relay and decoding a received signal may be impractical because the acquired CSI generally contains estimation errors because of noise, and also it can be outdated because of a feedback delay time. Therefore, performance evaluation and system design considering the impacts of both channel estimation errors and outdated CSI may be critical and necessary to satisfy performance requirements in practical communication environments. In [9], the outage probability and the average error rate of decode-and-forward (DF) relaying systems with BRS were investigated under identically distributed Rayleigh fading channels in the presence of both feedback delay and 


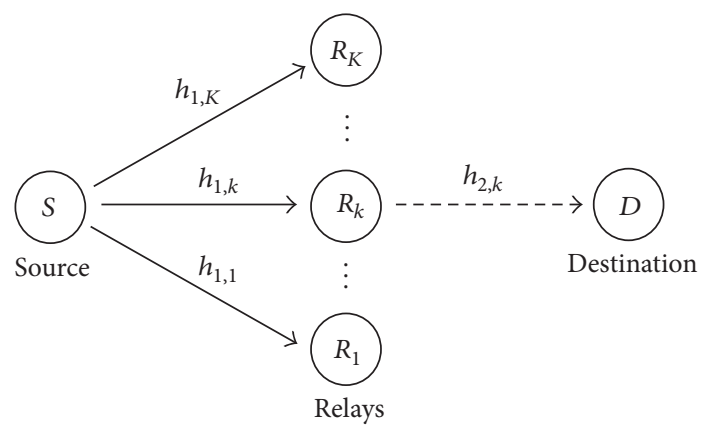

$\longrightarrow$ The first-hop transmission

$-\rightarrow$ The second-hop transmission

FIGURE 1: Dual-hop relaying system using relay selection where relay $k$ is selected.

channel estimation errors. In $[10,11]$, the outage probability and the average error rate of amplify-and-forward relaying systems with PRS and BRS were presented for identically distributed Rayleigh fading channels considering feedback delay. In [9-11], fading channels for all the links of each hop are assumed to be statistically identical. However, when relays are distributed over a sufficiently large area, the assumption of identically distributed fading channels can be impractical. In this paper, hence, we assume nonidentically distributed fading channels for all the links. In addition, to the best of our knowledge, outage performances of PRS and EPRS in DF relaying systems have not been studied in the presence of both feedback delay and channel estimation errors. Therefore, this paper focuses on investigation into the impact of both feedback delay and the channel estimation errors on the outage performances of PRS and EPRS in DF relaying systems. Considering outdated CSI and imperfect channel estimation with a DF relaying strategy, exact outage probabilities and asymptotic outage probabilities for PRS and EPRS are provided in closed form under independent and nonidentically distributed Rayleigh fading channels. Numerical investigations verify that the analytic results are perfectly matched with the simulated ones and show how much performance is degraded by the channel estimation errors and the feedback delay that induces the outdated CSI.

\section{System Model}

We consider a dual-hop DF relaying system using relay selection that consists of a source, $K$ relays, and a destination, as shown in Figure 1. All nodes are equipped with a single antenna. In this paper, we assume that the direct link between the source and the destination is unavailable due to high path loss and shadowing effect. The DF relays operate in a halfduplex mode, and PRS and EPRS are employed to select a single relay. As a relay selection protocol, proactive protocol [3] is considered, where a single relay is selected before data transmission. Because only the selected relay tries to receive and decode the signal from the source, the proactive protocol is more energy-efficient than reactive protocol, in which a single relay is selected after decoding the received signal at all relays. In the proactive protocol with DF relaying, the selected relay reencodes the decoded signal and forwards it to the destination only when the decoding succeeds.

Let the channel coefficient for relay $k(k \in\{1, \ldots, K\})$ at hop $i(i \in\{1,2\})$ be denoted as $h_{i, k}$, where the channels for relay $k$ at the first and the second hops mean those between the source and relay $k$ and between relay $k$ and the destination, respectively. The channels $h_{i, k}$ 's are assumed to be independent and nonidentically distributed complex Gaussian random variables with zero mean and variance $\beta_{i, k}$; that is, $h_{i, k} \sim \mathscr{C} \mathscr{N}\left(0, \beta_{i, k}\right)$. Also, considering block fading, the channels are assumed to be constant during a block length.

2.1. Channel Estimation Errors and Feedback Delay. The source and the relays transmit training signals orthogonally, and then the minimum-mean-square-error- (MMSE-) estimated channels at the relays and the destination are given by [12-14]

$$
h_{i, k}=\widehat{h}_{i, k}+e_{i, k}
$$

where $e_{i, k} \sim \mathscr{C} \mathcal{N}\left(0, \sigma_{e}^{2}\right)$ denotes the estimation error and $\widehat{h}_{i, k} \sim \mathscr{C} \mathscr{N}\left(0, \beta_{i, k}-\sigma_{e}^{2}\right)$ represents the MMSE-estimated channel. In this paper, we assume that the channel estimation error is independent of the received signal-to-noise ratio (SNR) for a data signal, and the estimation error distribution is identical at all the relays and the destination.

Since the estimated CSI is fed back for relay selection, the CSI may be outdated when the source and the selected relay transmit a data signal. Letting $\widehat{\beta}_{i, k} \triangleq \beta_{i, k}-\sigma_{e}^{2}$, the relation between the previously estimated channel $\widehat{h}_{i, k}$ and the currently estimated channel $\widetilde{h}_{i, k}$ can be modelled as $[13,15]$

$$
\tilde{h}_{i, k}=\rho_{d} \widehat{h}_{i, k}+\sqrt{1-\rho_{d}^{2}} u_{i, k}
$$

where $u_{i, k} \sim \mathscr{C} \mathscr{N}\left(0, \widehat{\beta}_{i, k}\right), \widetilde{h}_{i, k} \sim \mathscr{C} \mathscr{N}\left(0, \widehat{\beta}_{i, k}\right)$, and $\rho_{d}$ denotes the correlation coefficient between $\widehat{h}_{i, k}$ and $\widetilde{h}_{i, k}$, which can be defined as $\rho_{d} \triangleq J_{0}\left(2 \pi f_{d} \tau\right)[16] . J_{0}(\cdot)$ is a Bessel function of the first kind of zero order, $f_{d}$ is the maximum Doppler frequency, and $\tau$ is a feedback delay time. It is noted that $\widehat{h}_{i, k}$ and $\widetilde{h}_{i, k}$ are the channels used for relay selection and decoding, respectively. Hereafter, let $\widehat{g}_{i, k} \triangleq\left|\widehat{h}_{i, k}\right|^{2}$ and $\widetilde{g}_{i, k} \triangleq$ $\left|\widetilde{h}_{i, k}\right|^{2}$.

2.2. Partial Relay Selection Schemes. For PRS, the first-hop CSI is used to select a single relay. Thus, each relay feeds back the estimated CSI for the first hop to the source. Then, the source broadcasts the index of a selected relay to all the relays. The relay selected by PRS is expressed as [5]

$$
k_{p}^{*}=\arg \max _{k=1, \ldots, K}\left\{\widehat{g}_{1, k}\right\} .
$$

For EPRS, the first-hop estimated CSI for relay $k$ is used if $\widehat{\beta}_{1, k}<\widehat{\beta}_{2, k}$; otherwise the second-hop estimated CSI for relay $k$ is used. Every relay can know the average channel powers for the first and the second hops using the received 
signals from the source and the destination. Thus, each relay determines either to feed back the estimated CSI for the first hop to the destination or transmit the training signals to the destination for the second-hop channel estimation. Then, the destination selects a single relay and broadcasts the index of a selected relay to all the relays. The relay selected by EPRS is expressed as [7]

$$
k_{e}^{*}=\arg \max _{k=1, \ldots, K}\left\{\widehat{w}_{k}\right\}
$$

where $\widehat{w}_{k}=\widehat{g}_{1, k}$ for $\widehat{\beta}_{1, k}<\widehat{\beta}_{2, k}$; otherwise $\widehat{w}_{k}=\widehat{g}_{2, k}$.

\section{Outage Performance Analysis}

3.1. Received SNR with Channel Estimation Error. In this paper, we assume equal transmit powers at the source and the relays and equal noise powers at the relays and the destination, denoted as $P$ and $\sigma_{n}^{2}$, respectively, but it is straightforward to extend to different transmit powers. When the source or the selected relay (that succeeds in decoding) transmits $x$, the received signal at the relay or the destination is given by

$$
r_{i, k}=h_{i, k} \sqrt{P} x+n_{i, k}=\widetilde{h}_{i, k} \sqrt{P} x+e_{i, k}^{d} \sqrt{P} x+n_{i, k}
$$

where $E\left[|x|^{2}\right]=1, n_{i, k} \sim \mathscr{C} \mathscr{N}\left(0, \sigma_{n}^{2}\right)$ is an additive white Gaussian noise, and $e_{i, k}^{d} \sim \mathscr{C} \mathscr{N}\left(0, \sigma_{e}^{2}\right)$ denotes the error of MMSE-estimated channel in decoding. Let $\rho_{t} \triangleq P / \sigma_{n}^{2}$ and $\zeta \triangleq \rho_{t} /\left(1+\rho_{t} \sigma_{e}^{2}\right)$, where $\rho_{t}$ denotes the average transmit SNR. Then the received SNR with channel estimation error is obtained as $\widetilde{\gamma}_{i, k}=\zeta \widetilde{g}_{i, k}$, where it is noted that $\widetilde{h}_{i, k}$ is used instead of $\widehat{h}_{i, k}$ because of the received signal for decoding, not relay selection. It is also noted that there is no received signal at the destination when the selected relay fails to decode the signal received from the source.

3.2. Exact Outage Probability Analysis. An outage probability expression of PRS can be easily obtained from that of EPRS. Thus, in this paper, we show only derivations of outage probability of EPRS.

Using [17, equations (2) and (3)] and (4), for a given target data rate $R$ in bps/Hz, the outage probability of EPRS in dual-hop DF relaying systems in the presence of channel estimation error and feedback delay is obtained by

$$
\begin{aligned}
P_{o}(R) & =\sum_{k=1}^{K} \operatorname{Pr}\left\{\frac{1}{2} \log _{2}\left(1+\widetilde{\gamma}_{2, k}\right)\right. \\
& \left.<R, \frac{1}{2} \log _{2}\left(1+\widetilde{\gamma}_{1, k}\right)>R, \widehat{w}_{k}>\max _{\substack{j=1, \ldots, K \\
j \neq k}}\left\{\widehat{w}_{j}\right\}\right\}
\end{aligned}
$$

$$
\begin{aligned}
& +\sum_{k=1}^{K} \operatorname{Pr}\left\{\frac{1}{2} \log _{2}\left(1+\widetilde{\gamma}_{1, k}\right)<R, \widehat{w}_{k}\right. \\
& \left.>\max _{\substack{j=1, \ldots, K \\
j \neq k}}\left\{\widehat{w}_{j}\right\}\right\},
\end{aligned}
$$

where it is assumed that the decoding at relay $k$ succeeds when the achievable data rate between the source and relay $k$ exceeds $R$ [17]. In (6), the first and the second parts mean the outage probability in case of decoding success and failure at the selected relay, respectively. Letting $R_{t} \triangleq\left(2^{2 R}-1\right) / \zeta,(6)$ can be rewritten as

$$
\begin{aligned}
& P_{o}(R) \\
& =\sum_{k=1}^{K} \operatorname{Pr}\left\{\widetilde{g}_{2, k}<R_{t}, \widetilde{g}_{1, k}>R_{t}, \widehat{w}_{k}>\max _{\substack{j=1, \ldots, K \\
j \neq k}}\left\{\widehat{w}_{j}\right\}\right\} \\
& +\sum_{k=1}^{K} \operatorname{Pr}\left\{\widetilde{g}_{1, k}<R_{t}, \widehat{w}_{k}>\max _{\substack{j=1, \ldots, K \\
j \neq k}}\left\{\widehat{w}_{j}\right\}\right\} .
\end{aligned}
$$

If $\widehat{w}_{k}=\widehat{g}_{1, k}$, then the first and the second parts in (7) are, respectively, expressed as

$$
\begin{gathered}
\operatorname{Pr}\left\{\widetilde{g}_{2, k}<R_{t}\right\} \operatorname{Pr}\left\{\widetilde{g}_{1, k}>R_{t}, \widehat{g}_{1, k}>\max _{\substack{j=1, \ldots, K \\
j \neq k}}\left\{\widehat{w}_{j}\right\}\right\}, \\
\operatorname{Pr}\left\{\tilde{g}_{1, k}<R_{t}, \widehat{g}_{1, k}>\max _{\substack{j=1, \ldots, K \\
j \neq k}}\left\{\widehat{w}_{j}\right\}\right\} .
\end{gathered}
$$

On the other hand, if $\widehat{w}_{k}=\widehat{g}_{2, k}$, then the first and the second parts in (7) are expressed as

$$
\begin{gathered}
\operatorname{Pr}\left\{\widetilde{g}_{1, k}>R_{t}\right\} \operatorname{Pr}\left\{\widetilde{g}_{2, k}<R_{t}, \widehat{g}_{2, k}>\max _{\substack{j=1, \ldots, K \\
j \neq k}}\left\{\widehat{w}_{j}\right\}\right\}, \\
\operatorname{Pr}\left\{\widetilde{g}_{1, k}<R_{t}\right\} \operatorname{Pr}\left\{\widehat{g}_{2, k}>\max _{\substack{j=1, \ldots, K \\
j \neq k}}\left\{\widehat{w}_{j}\right\}\right\} .
\end{gathered}
$$

Conditioned on $\widehat{g}_{i, k}=z, \widetilde{g}_{i, k}$ is a noncentral Chi-square distributed random variable with two degrees of freedom, and its probability density function (PDF) is obtained using [18, eq. (2-1-118)] as follows:

$$
\begin{gathered}
\operatorname{Pr}\left\{\widetilde{g}_{i, k}=x \mid \widehat{g}_{i, k}=z\right\}=\frac{1}{\widehat{\beta}_{i, k}\left(1-\rho_{d}^{2}\right)} \\
\cdot e^{-\left(\rho_{d}^{2} z+x\right) /\left(\widehat{\beta}_{i, k}\left(1-\rho_{d}^{2}\right)\right)} I_{0}\left(\sqrt{\frac{4 \rho_{d}^{2} z x}{\widehat{\beta}_{i, k}^{2}\left(1-\rho_{d}^{2}\right)^{2}}}\right),
\end{gathered}
$$


where $I_{0}(\cdot)$ is a modified Bessel function of the first kind of order zero. Using [18, eq. (2-1-120)], (12) is rewritten as

$$
\begin{aligned}
& \operatorname{Pr}\left\{\tilde{g}_{i, k}=x \mid \hat{g}_{i, k}=z\right\}=\frac{1}{\widehat{\beta}_{i, k}\left(1-\rho_{d}^{2}\right)} \\
& \cdot e^{-\left(\rho_{d}^{2} z+x\right) /\left(\widehat{\beta}_{i, k}\left(1-\rho_{d}^{2}\right)\right)} \sum_{m=0}^{\infty} \frac{1}{(m !)^{2}}\left(\frac{\rho_{d}^{2} z x}{\hat{\beta}_{i, k}^{2}\left(1-\rho_{d}^{2}\right)^{2}}\right)^{m} .
\end{aligned}
$$

Using (13), the cumulative distribution function (CDF) and complementary CDF of $\tilde{g}_{i, k}$ conditioned on $\hat{g}_{i, k}=z$ are, respectively, obtained by

$$
\begin{aligned}
& \operatorname{Pr}\left\{\tilde{g}_{i, k}<x \mid \hat{g}_{i, k}=z\right\} \\
& =\int_{0}^{x} \operatorname{Pr}\left\{\widetilde{g}_{i, k}=y \mid \widehat{g}_{i, k}=z\right\} d y=\frac{1}{\widehat{\beta}_{i, k}\left(1-\rho_{d}^{2}\right)} \\
& \cdot e^{-\rho_{d}^{2} z /\left(\widehat{\beta}_{i, k}\left(1-\rho_{d}^{2}\right)\right)} \sum_{m=0}^{\infty} \frac{1}{(m !)^{2}}\left(\frac{\rho_{d}^{2} z}{\widehat{\beta}_{i, k}\left(1-\rho_{d}^{2}\right)}\right)^{m} \\
& \cdot \int_{0}^{x}\left(\frac{y}{\widehat{\beta}_{i, k}\left(1-\rho_{d}^{2}\right)}\right)^{m} e^{-y /\left(\widehat{\beta}_{i, k}\left(1-\rho_{d}^{2}\right)\right)} d y \\
& =e^{-\rho_{d}^{2} z /\left(\widehat{\beta}_{i, k}\left(1-\rho_{d}^{2}\right)\right)} \sum_{m=0}^{\infty} \frac{1}{(m !)^{2}}\left(\frac{\rho_{d}^{2} z}{\widehat{\beta}_{i, k}\left(1-\rho_{d}^{2}\right)}\right)^{m} \\
& \cdot \gamma\left(m+1, \frac{x}{\widehat{\beta}_{i, k}\left(1-\rho_{d}^{2}\right)}\right), \\
& \operatorname{Pr}\left\{\tilde{g}_{i, k}>x \mid \widehat{g}_{i, k}=z\right\} \\
& =\int_{x}^{\infty} \operatorname{Pr}\left\{\widetilde{g}_{i, k}=y \mid \widehat{g}_{i, k}=z\right\} d y=\frac{1}{\widehat{\beta}_{i, k}\left(1-\rho_{d}^{2}\right)} \\
& \cdot e^{-\rho_{d}^{2} z /\left(\widehat{\beta}_{i, k}\left(1-\rho_{d}^{2}\right)\right)} \sum_{m=0}^{\infty} \frac{1}{(m !)^{2}}\left(\frac{\rho_{d}^{2} z}{\widehat{\beta}_{i, k}\left(1-\rho_{d}^{2}\right)}\right)^{m} \\
& \cdot \int_{x}^{\infty}\left(\frac{y}{\widehat{\beta}_{i, k}\left(1-\rho_{d}^{2}\right)}\right)^{m} e^{-y /\left(\widehat{\beta}_{i, k}\left(1-\rho_{d}^{2}\right)\right)} d y \\
& =e^{-\rho_{d}^{2} z /\left(\widehat{\beta}_{i, k}\left(1-\rho_{d}^{2}\right)\right)} \sum_{m=0}^{\infty} \frac{1}{(m !)^{2}}\left(\frac{\rho_{d}^{2} z}{\widehat{\beta}_{i, k}\left(1-\rho_{d}^{2}\right)}\right)^{m} \\
& \cdot\left(m !-\gamma\left(m+1, \frac{x}{\widehat{\beta}_{i, k}\left(1-\rho_{d}^{2}\right)}\right)\right),
\end{aligned}
$$

where $\gamma(\cdot, \cdot)$ denotes the incomplete gamma function and we used $\int_{x}^{\infty} t^{m-1} e^{-t} d t=(m-1) !-\gamma(m, x)$ to derive the complementary CDF.
Let $\hat{y}_{k} \triangleq \max _{j=1, \ldots, K, j \neq k}\left\{\widehat{w}_{j}\right\}$. Then, the CDF of $\hat{y}_{k}$ is obtained by

$$
\begin{aligned}
\operatorname{Pr}\left\{\widehat{y}_{k}<x\right\} & =\prod_{j=1, j \neq k}^{K} \operatorname{Pr}\left\{\widehat{w}_{j}<x\right\}=\prod_{j=1, j \neq k}^{K}\left(1-e^{-x / \lambda_{j}}\right) \\
& =1+\sum_{j=1}^{K-1} \sum_{L_{j} \leq S_{k}}(-1)^{j} e^{-x \sum_{q \in L_{j}} 1 / \lambda_{q}},
\end{aligned}
$$

where $S_{k}=\{1, \ldots, k-1, k+1, \ldots, K\}, L_{j}$ represents all possible subsets of $S_{k}$ with the cardinality of $j$, and $\lambda_{q}=\widehat{\beta}_{1, q}$ if $\widehat{\beta}_{1, q}<\widehat{\beta}_{1, q}$; otherwise $\lambda_{q}=\widehat{\beta}_{2, q}$.

Using (15), (16), and $\operatorname{Pr}\left\{\hat{g}_{i, k}=z\right\}=\left(1 / \widehat{\beta}_{i, k}\right) e^{-z / \widehat{\beta}_{i, k}}$, $\operatorname{Pr}\left\{\widetilde{g}_{i, k}>R_{t}, \widehat{g}_{i, k}>\max _{j=1, \ldots, K, j \neq k}\left\{\widehat{w}_{j}\right\}\right\}$ in (8) is derived as follows:

$$
\begin{aligned}
\int_{0}^{\infty} & \operatorname{Pr}\left\{\widetilde{g}_{i, k}>R_{t} \mid \widehat{g}_{i, k}=z\right\} \operatorname{Pr}\left\{\widehat{y}_{k}<z \mid \widehat{g}_{i, k}=z\right\} \operatorname{Pr}\left\{\widehat{g}_{i, k}\right. \\
= & z\} d z \\
& =\int_{0}^{\infty}\left[e^{-\rho_{d}^{2} z /\left(\widehat{\beta}_{i, k}\left(1-\rho_{d}^{2}\right)\right)} \sum_{m=0}^{\infty} \frac{1}{(m !)^{2}}\left(\frac{\rho_{d}^{2} z}{\widehat{\beta}_{i, k}\left(1-\rho_{d}^{2}\right)}\right)^{m}\right. \\
& \cdot\left(m !-\gamma\left(m+1, \frac{R_{t}}{\hat{\beta}_{i, k}\left(1-\rho_{d}^{2}\right)}\right)\right) \\
& \left.\cdot\left(1+\sum_{j=1}^{K-1} \sum_{L_{j} \leq S_{k}}(-1)^{j} e^{-z \sum_{q \in L_{j}} 1 / \lambda_{q}}\right) \frac{1}{\widehat{\beta}_{i, k}} e^{-z / \widehat{\beta}_{i, k}}\right] d z \\
= & \sum_{m=0}^{\infty}\left(m !-\gamma\left(m+1, \frac{R_{t}}{\widehat{\beta}_{i, k}\left(1-\rho_{d}^{2}\right)}\right)\right) \\
& \cdot \frac{\left(\rho_{d}^{2}\right)^{m}\left(1-\rho_{d}^{2}\right)}{m !} \\
& +\sum_{j=1}^{K-1} \sum_{L_{j} \leq S_{k}} \sum_{m=0}^{\infty}\left(m !-\gamma\left(m+1, \frac{R_{t}}{\widehat{\hat{\beta}}_{i, k}\left(1-\rho_{d}^{2}\right)}\right)\right) \\
& \cdot \frac{(-1)^{j}}{m !}\left(\frac{\rho_{d}^{2}}{1-\rho_{d}^{2}}\right)^{m}\left(\frac{1}{1-\rho_{d}^{2}}+\widehat{\beta}_{i, k} \sum_{q \in L_{j}} \frac{1}{\lambda_{q}}\right)^{-m-1} .
\end{aligned}
$$

Analogous to (17), using (14), (16), and $\operatorname{Pr}\left\{\hat{g}_{i, k}=z\right\}=$ $\left(1 / \widehat{\beta}_{i, k}\right) e^{-z / \widehat{\beta}_{i, k}}, \operatorname{Pr}\left\{\widetilde{g}_{i, k}<R_{t}, \widehat{g}_{i, k}>\max _{j=1, \ldots, K, j \neq k}\left\{\widehat{w}_{j}\right\}\right\}$ in (9) and (10) is derived as follows:

$$
\begin{aligned}
\int_{0}^{\infty} & \operatorname{Pr}\left\{\widetilde{g}_{i, k}<R_{t} \mid \hat{g}_{i, k}=z\right\} \operatorname{Pr}\left\{\hat{y}_{k}<z \mid \hat{g}_{i, k}=z\right\} \operatorname{Pr}\left\{\hat{g}_{i, k}\right. \\
= & z\} d z \\
& =\int_{0}^{\infty}\left[e^{-\rho_{d}^{2} z /\left(\widehat{\beta}_{i, k}\left(1-\rho_{d}^{2}\right)\right)} \sum_{m=0}^{\infty} \frac{1}{(m !)^{2}}\left(\frac{\rho_{d}^{2} z}{\widehat{\beta}_{i, k}\left(1-\rho_{d}^{2}\right)}\right)^{m}\right. \\
& \cdot \gamma\left(m+1, \frac{R_{t}}{\hat{\beta}_{i, k}\left(1-\rho_{d}^{2}\right)}\right)
\end{aligned}
$$




$$
\begin{aligned}
& \left.\cdot\left(1+\sum_{j=1}^{K-1} \sum_{L_{j} \subseteq S_{k}}(-1)^{j} e^{-z \sum_{q \in L_{j}} 1 / \lambda_{q}}\right) \frac{1}{\widehat{\beta}_{i, k}} e^{-z / \widehat{\beta}_{i, k}}\right] d z \\
& =\sum_{m=0}^{\infty} \gamma\left(m+1, \frac{R_{t}}{\widehat{\beta}_{i, k}\left(1-\rho_{d}^{2}\right)}\right) \frac{\left(\rho_{d}^{2}\right)^{m}\left(1-\rho_{d}^{2}\right)}{m !} \\
& +\sum_{j=1}^{K-1} \sum_{L_{j} \subseteq S_{k}} \sum_{m=0}^{\infty} \gamma\left(m+1, \frac{R_{t}}{\widehat{\beta}_{i, k}\left(1-\rho_{d}^{2}\right)}\right) \frac{(-1)^{j}}{m !} \\
& \cdot\left(\frac{\rho_{d}^{2}}{1-\rho_{d}^{2}}\right)^{m}\left(\frac{1}{1-\rho_{d}^{2}}+\widehat{\beta}_{i, k} \sum_{q \in L_{j}} \frac{1}{\lambda_{q}}\right)^{-m-1} .
\end{aligned}
$$

Then, using (16), $\operatorname{Pr}\left\{\widehat{g}_{2, k}>\max _{j=1, \ldots, K, j \neq k}\left\{\widehat{w}_{j}\right\}\right\}$ in (11) is obtained as follows:

$$
\begin{aligned}
& \int_{0}^{\infty} \operatorname{Pr}\left\{\widehat{y}_{k}<z \mid \widehat{g}_{2, k}=z\right\} \operatorname{Pr}\left\{\widehat{g}_{2, k}=z\right\} d z \\
& =\int_{0}^{\infty}\left(1+\sum_{j=1}^{K-1} \sum_{L_{j} \subseteq S_{k}}(-1)^{j} e^{-z \sum_{q \in L_{j}} 1 / \lambda_{q}}\right) \\
& \cdot \frac{1}{\widehat{\beta}_{2, k}} e^{-z / \widehat{\beta}_{2, k}} d z=1 \\
& +\sum_{j=1}^{K-1} \sum_{L_{j} \subseteq S_{k}} \frac{(-1)^{j}}{\widehat{\beta}_{2, k}}\left(\frac{1}{\widehat{\beta}_{2, k}}+\sum_{q \in L_{j}} \frac{1}{\lambda_{q}}\right)^{-1} .
\end{aligned}
$$

Finally, we obtain the outage probability by substituting (17)(19), $\operatorname{Pr}\left\{\widetilde{g}_{i, k}<R_{t}\right\}=1-e^{-R_{t} / \widehat{\beta}_{i, k}}$, and $\operatorname{Pr}\left\{\widetilde{g}_{i, k}>R_{t}\right\}=e^{-R_{t} / \widehat{\beta}_{i, k}}$ into (8)-(11) and inserting (8)-(11) into (7). Also, the outage probability of PRS can be easily obtained by inserting (17) and (18) with $\lambda_{q}=\widehat{\beta}_{1, q}$ into (8) and (9), respectively, and substituting (8) and (9) into (7).

3.3. Asymptotic Outage Probability Analysis. In this section, different asymptotic analysis is performed according to the condition of $\sigma_{e}^{2}$ because when $\sigma_{e}^{2}=0$ (i.e., perfect channel estimation), $R_{t}=\left(2^{2 R}-1\right) / \rho_{t}$, whereas when $\sigma_{e}^{2} \neq 0$, $R_{t}=\left(2^{2 R}-1\right)\left(1+\rho_{t} \sigma_{e}^{2}\right) / \rho_{t} \approx\left(2^{2 R}-1\right) \sigma_{e}^{2}$ by high SNR approximation. It is noted that when $\sigma_{e}^{2} \neq 0, R_{t}$ does not depend upon $\rho_{t}$.

When $\sigma_{e}^{2}=0$, using $R_{t}=\left(2^{2 R}-1\right) / \rho_{t}$ and high SNR approximation, the following approximated equation is obtained:

$$
\begin{aligned}
& \gamma\left(m+1, \frac{R_{t}}{\widehat{\beta}_{i, k}\left(1-\rho_{d}^{2}\right)}\right) \\
& \quad \gamma\left(m+1, \frac{2^{2 R}-1}{\rho_{t} \widehat{\beta}_{i, k}\left(1-\rho_{d}^{2}\right)}\right) \\
& \stackrel{\rho_{t} \rightarrow \infty}{\approx} \frac{1}{m+1}\left(\frac{2^{2 R}-1}{\rho_{t} \widehat{\beta}_{i, k}\left(1-\rho_{d}^{2}\right)}\right)^{m+1} .
\end{aligned}
$$

Using (20), (17) and (18) are, respectively, approximated as

$$
\begin{aligned}
& \sum_{m=0}^{\infty}\left(\rho_{d}^{2}\right)^{m}\left(1-\rho_{d}^{2}\right)+\sum_{j=1}^{K-1} \sum_{L_{j} \subseteq S_{k}} \sum_{m=0}^{\infty}(-1)^{j}\left(\frac{\rho_{d}^{2}}{1-\rho_{d}^{2}}\right)^{m} \\
& \cdot\left(\frac{1}{1-\rho_{d}^{2}}+\widehat{\beta}_{i, k} \sum_{q \in L_{j}} \frac{1}{\lambda_{q}}\right)^{-m-1}, \\
& \frac{2^{2 R}-1}{\rho_{t} \widehat{\beta}_{i, k}}\left\{1+\left(\frac{1}{1-\rho_{d}^{2}}\right) \sum_{j=1}^{K-1} \sum_{L_{j} \subseteq S_{k}}(-1)^{j}\right. \\
& \left.\cdot\left(\frac{1}{1-\rho_{d}^{2}}+\widehat{\beta}_{i, k} \sum_{q \in L_{j}} \frac{1}{\lambda_{q}}\right)^{-1}\right\} .
\end{aligned}
$$

Using (21), (22), $\operatorname{Pr}\left\{\tilde{g}_{i, k}<R_{t}\right\} \approx\left(2^{2 R}-1\right) /\left(\rho_{t} \widehat{\beta}_{i, k}\right)$, and $\operatorname{Pr}\left\{\widetilde{g}_{i, k}>R_{t}\right\} \approx 1$ by high SNR approximation, (8)-(11) are, respectively, approximated as

$$
\begin{aligned}
& \left(\frac{2^{2 R}-1}{\rho_{t} \widehat{\beta}_{2, k}}\right)\left\{\sum_{m=0}^{\infty}\left(\rho_{d}^{2}\right)^{m}\left(1-\rho_{d}^{2}\right)\right. \\
& +\sum_{j=1}^{K-1} \sum_{L_{j} \subseteq S_{k}} \sum_{m=0}^{\infty}(-1)^{j}\left(\frac{\rho_{d}^{2}}{1-\rho_{d}^{2}}\right)^{m} \\
& \left.\cdot\left(\frac{1}{1-\rho_{d}^{2}}+\widehat{\beta}_{1, k} \sum_{q \in L_{j}} \frac{1}{\lambda_{q}}\right)^{-m-1}\right\} \text {, } \\
& \left(\frac{2^{2 R}-1}{\rho_{t} \widehat{\beta}_{1, k}}\right)\left\{1+\left(\frac{1}{1-\rho_{d}^{2}}\right)\right. \\
& \left.\cdot \sum_{j=1}^{K-1} \sum_{L_{j} \subseteq S_{k}}(-1)^{j}\left(\frac{1}{1-\rho_{d}^{2}}+\widehat{\beta}_{1, k} \sum_{q \in L_{j}} \frac{1}{\lambda_{q}}\right)^{-1}\right\}, \\
& \left(\frac{2^{2 R}-1}{\rho_{t} \widehat{\beta}_{2, k}}\right)\left\{1+\left(\frac{1}{1-\rho_{d}^{2}}\right)\right. \\
& \left.\cdot \sum_{j=1}^{K-1} \sum_{L_{j} \subseteq S_{k}}(-1)^{j}\left(\frac{1}{1-\rho_{d}^{2}}+\widehat{\beta}_{2, k} \sum_{q \in L_{j}} \frac{1}{\lambda_{q}}\right)^{-1}\right\}, \\
& \left(\frac{2^{2 R}-1}{\rho_{t} \widehat{\beta}_{1, k}}\right)\{1 \\
& \left.+\sum_{j=1}^{K-1} \sum_{L_{j} \subseteq S_{k}} \frac{(-1)^{j}}{\widehat{\beta}_{2, k}}\left(\frac{1}{\widehat{\beta}_{2, k}}+\sum_{q \in L_{j}} \frac{1}{\lambda_{q}}\right)^{-1}\right\} .
\end{aligned}
$$

Finally, the asymptotic outage probability of EPRS for $\sigma_{e}^{2}=$ 0 can be obtained by substituting (23)-(26) into (7). In addition, the asymptotic outage probability of PRS for $\sigma_{e}^{2}=$ 0 can be obtained by inserting (23) and (24) into (7). 
TABLE 1: Description of simulation cases.

\begin{tabular}{|c|c|c|c|}
\hline Cases & $R$ & K & $\begin{array}{c}\text { Average channel } \\
\text { powers }\end{array}$ \\
\hline I & $1 \mathrm{bps} / \mathrm{Hz}$ & 2 & $\begin{array}{c}\beta_{1,1}=1.1 \\
\beta_{1,2}=12 \\
\beta_{2,1}=11 \\
\beta_{2,2}=1.2\end{array}$ \\
\hline II & $1 \mathrm{bps} / \mathrm{Hz}$ & 3 & $\begin{array}{l}\beta_{1,1}=1.1 \\
\beta_{1,2}=12 \\
\beta_{1,3}=1.3 \\
\beta_{2,1}=11 \\
\beta_{2,2}=1.2 \\
\beta_{2,3}=13\end{array}$ \\
\hline III & $1 \mathrm{bps} / \mathrm{Hz}$ & 3 & $\begin{array}{l}\beta_{1,1}=1.1 \\
\beta_{1,2}=4.8 \\
\beta_{1,3}=1.3 \\
\beta_{2,1}=4.4 \\
\beta_{2,2}=1.2 \\
\beta_{2,3}=5.2\end{array}$ \\
\hline
\end{tabular}

From (23)-(26), it is observed that the order of $1 / \rho_{t}$ in the asymptotic outage probability expression is one, and hence the diversity orders of EPRS and PRS are one.

In contrast to the asymptotic analysis for $\sigma_{e}^{2}=0$, the asymptotic outage probability of EPRS for $\sigma_{e}^{2} \neq 0$ is easily obtained by inserting $R_{t} \approx\left(2^{2 R}-1\right) \sigma_{e}^{2}$ in (8)-(11), (17), and (18). Also, the asymptotic outage probability of PRS for $\sigma_{e}^{2} \neq 0$ is simply obtained by replacing with $R_{t} \approx\left(2^{2 R}-1\right) \sigma_{e}^{2}$ in (8), (9), (17), and (18). Therefore, the asymptotic outage performance for EPRS and PRS is not affected by $\rho_{t}$, which results in the diversity order of zero.

\section{Numerical Results}

To verify the analysis presented in this paper and evaluate the outage performance, we consider three simulation cases as shown in Table 1 , where it is noted that $\widehat{\beta}_{i, k}$ 's in the outage probability expression are obtained by $\widehat{\beta}_{i, k}=\beta_{i, k}-\sigma_{e}^{2}$. In Cases I and II, $\left|10 \log _{10}\left(\beta_{1, k} / \beta_{2, k}\right)\right|=10 \mathrm{~dB}$ for all $k$, and $K=2$ and 3 , respectively, whereas in Case III, $\left|10 \log _{10}\left(\beta_{1, k} / \beta_{2, k}\right)\right|=6 \mathrm{~dB}$ for all $k$, and $K=3$, where $\left|10 \log _{10}\left(\beta_{1, k} / \beta_{2, k}\right)\right|$ means a difference between the average channel powers for the first and the second hops at relay $k$. It is noted that Cases I and II are better scenarios than Case III, since EPRS and PRS provide better outage performance as the gap between the average channel powers for the first and the second hops becomes larger. Table 2 illustrates the values of correlation coefficient $\rho_{d}=J_{0}\left(2 \pi f_{d} \tau\right)$ for various conditions. It is noted that the carrier frequency of $2.0 \mathrm{GHz}$ has been used for $3 \mathrm{GPP}$ LTE system simulation as shown in [19]. From Table 2, we choose $\rho_{d}=0.5,0.8,0.9$, and 1.0 for simulations, where $\rho_{d}=$ 1.0 means that there is no feedback delay. It is noted that for $\rho_{d}=1.0$ only simulated results are shown, since it is impossible to solve the outage probability expression when $\rho_{d}=1.0$. Figures $2-7$ show the outage probabilities of EPRS and PRS with various correlation coefficients (dependent

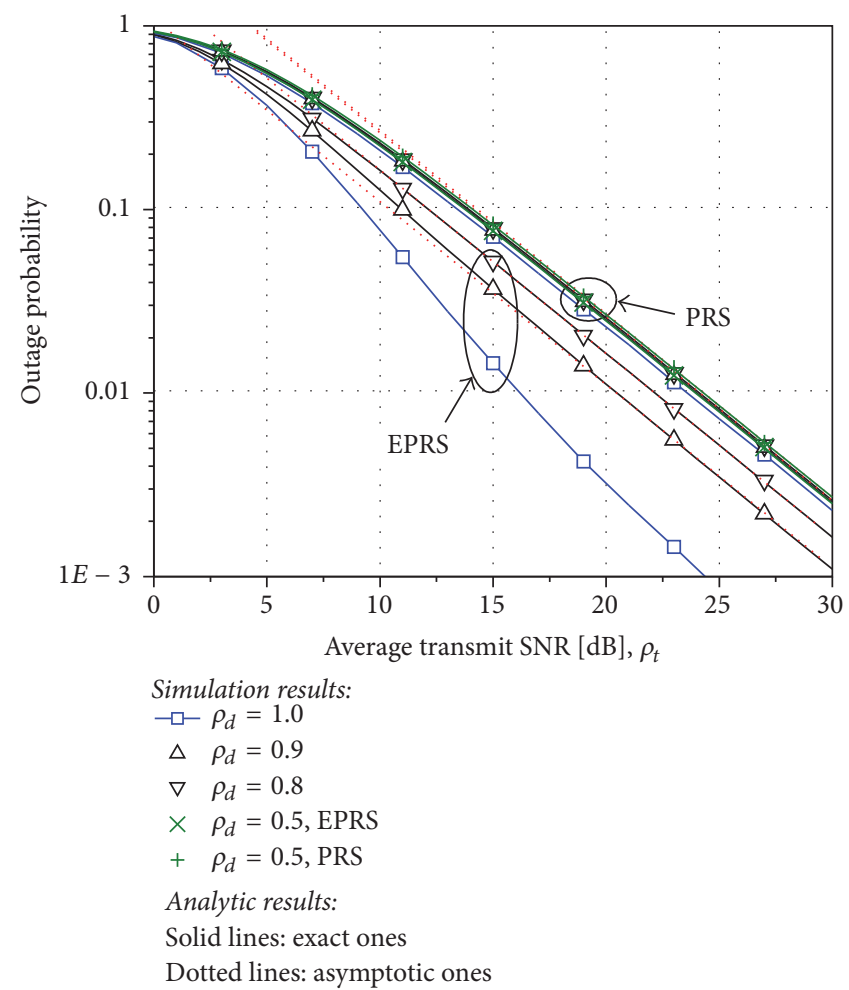

FIGURE 2: Outage probabilities of EPRS and PRS with perfect channel estimation for Case I when $\rho_{d}=0.5,0.8,0.9,1.0$.

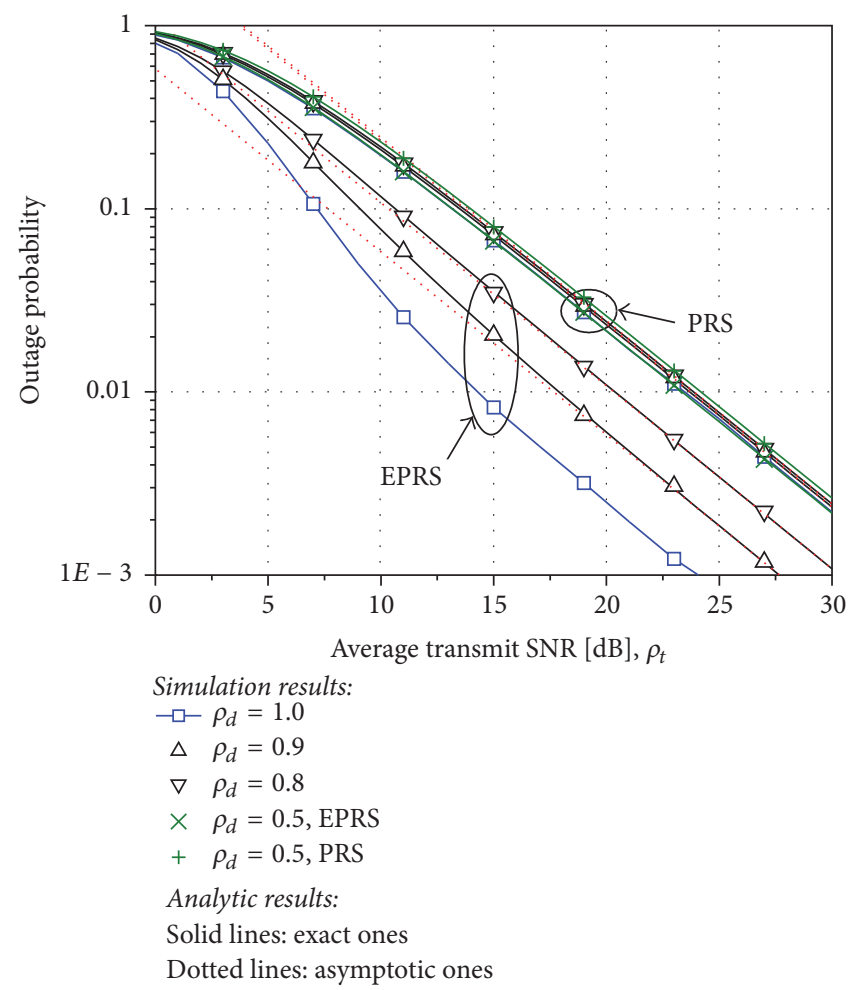

FIGURE 3: Outage probabilities of EPRS and PRS with perfect channel estimation for Case II when $\rho_{d}=0.5,0.8,0.9,1.0$.

on feedback delay time) and channel estimation errors for Cases I-III. All the figures demonstrate that the theoretical 
TABLE 2: Correlation coefficient $\rho_{d}=J_{0}\left(2 \pi f_{d} \tau\right)$, where $f_{c}$ denotes the carrier frequency.

\begin{tabular}{|c|c|c|c|c|c|}
\hline$f_{c}[\mathrm{GHz}]$ & $\tau[\mathrm{ms}]$ & $4.5 \mathrm{~km} / \mathrm{h}$ & $10 \mathrm{~km} / \mathrm{h}$ & $30 \mathrm{~km} / \mathrm{h}$ & $60 \mathrm{~km} / \mathrm{h}$ \\
\hline 2.0 & 0.5 & 0.9998 & 0.9992 & 0.9924 & 0.9698 \\
\hline 2.0 & 1 & 0.9993 & 0.9966 & 0.9698 & 0.8818 \\
\hline 2.0 & 1.5 & 0.9985 & 0.9924 & 0.9326 & 0.7441 \\
\hline 0.8 & 1.5 & 0.9998 & 0.9988 & 0.9891 & 0.9566 \\
\hline 3.0 & 1.5 & 0.9965 & 0.9829 & 0.8516 & 0.4720 \\
\hline
\end{tabular}

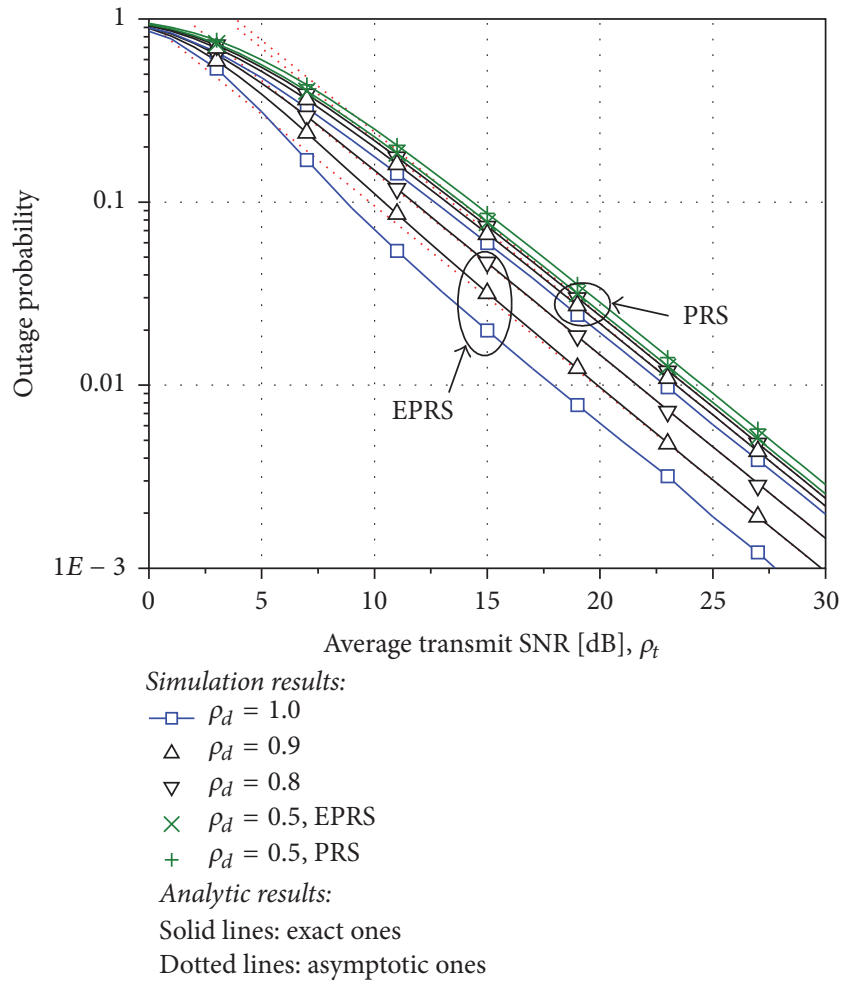

FIGURE 4: Outage probabilities of EPRS and PRS with perfect channel estimation for Case III when $\rho_{d}=0.5,0.8,0.9,1.0$.

analysis of exact outage probabilities is in perfect agreement with simulation results, and the asymptotic results are well matched with the simulated ones in the high SNR regime.

Figures 2-4 show the outage probabilities of EPRS and PRS with $\rho_{d}=0.5,0.8,0.9$, and 1.0 for Cases I-III, respectively, when $\sigma_{e}^{2}=0$ (i.e., perfect channel estimation). From the figures, it is observed that the outage performance of EPRS is significantly degraded as $\rho_{d}$ decreases (i.e., the feedback delay time increases), whereas the outage performance of PRS is nearly impervious to $\rho_{d}$. Also, it is indicated that the diversity orders of EPRS and PRS are not changed by $\rho_{d}$, since their diversity orders are one regardless of $\rho_{d}$. In comparing the outage performances for Cases I and II in Figures 2 and 3, respectively, EPRS achieves better outage performance than PRS as $K$ increases, but the outage performance of EPRS becomes closer to that of PRS as both $K$ and $\rho_{d}$ diminish. The reason is that partial CSI used for EPRS can well represent the end-to-end channel quality for high $\rho_{d}$, but the accuracy of the partial CSI becomes worse as $\rho_{d}$ decreases. In comparing

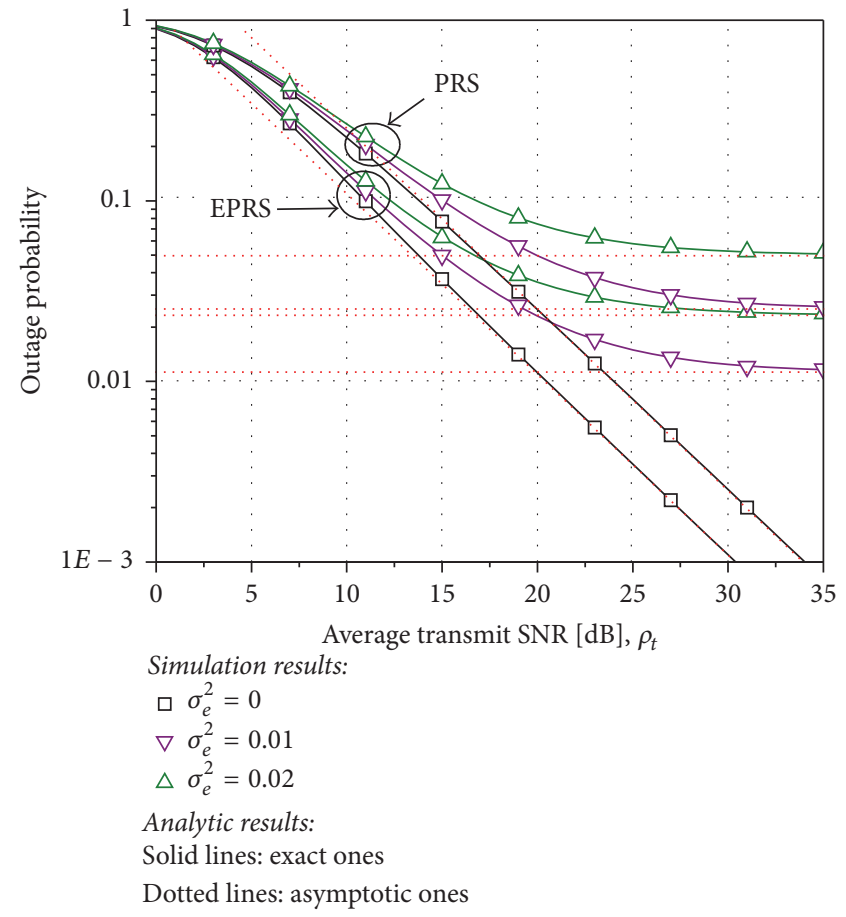

FIGURE 5: Outage probabilities of EPRS and PRS with $\rho_{d}=0.9$ for Case I when $\sigma_{e}^{2}=0,0.01,0.02$.

the outage performances for Cases II and III in Figures 3 and 4 , respectively, the outage performance of EPRS is degraded and is close to that of PRS as an average channel power gap between the first and the second hops decreases, since the accuracy of the partial CSI for EPRS is poor when an average channel power gap between the first and the second hops is small.

Figures 5-7 show the outage probabilities of EPRS and PRS with $\sigma_{e}^{2}=0,0.01$, and 0.02 for Cases I-III, respectively, when $\rho_{d}=0.9$. The figures indicate that the outage performances of both EPRS and PRS become worse and saturated as $\sigma_{e}^{2}$ increases, and their diversity orders are considerably reduced even for low $\sigma_{e}^{2}$. It is noted that, for $\sigma_{e}^{2} \neq 0$, all the asymptotic results are constant with respect to $\rho_{t}$; that is, the diversity orders are zero. In addition, it is remarkable that when $\sigma_{e}^{2}$ increases from 0.01 to 0.02 , a level of performance degradation of EPRS and PRS is similar for Cases I-III. In the outage performances for Cases I-III in Figures 5-7, respectively, analogous to the results in Figures 2-4, EPRS attains better performance than PRS as either $K$ or an average 


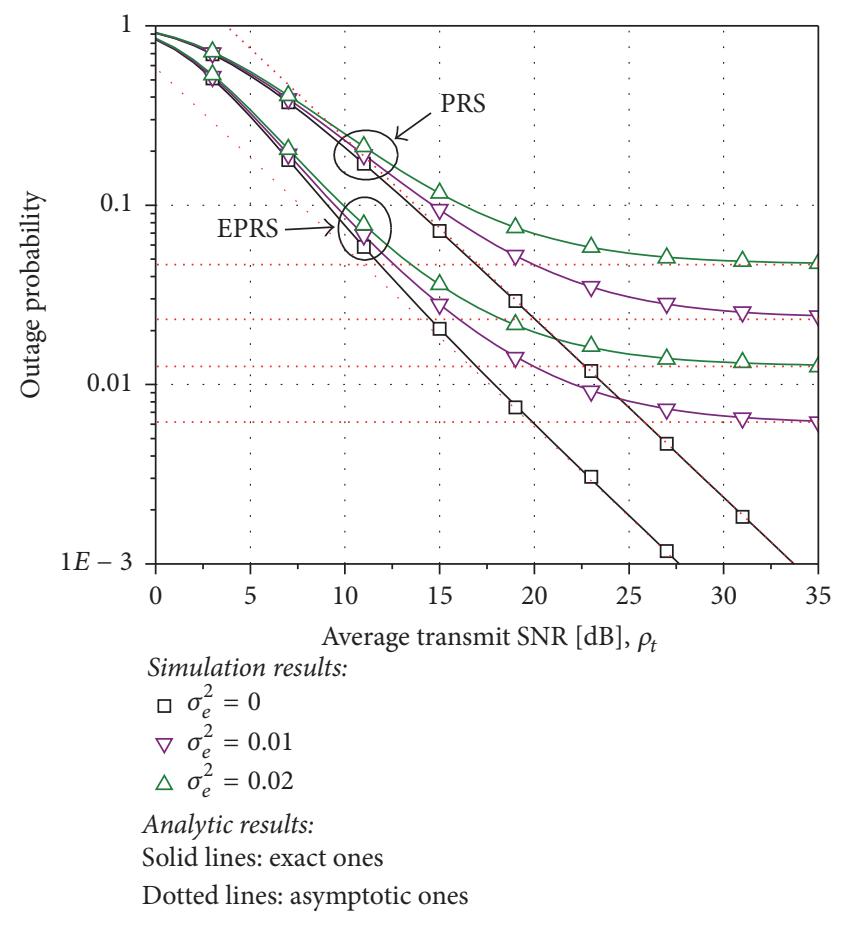

FIGURE 6: Outage probabilities of EPRS and PRS with $\rho_{d}=0.9$ for Case II when $\sigma_{e}^{2}=0,0.01,0.02$.

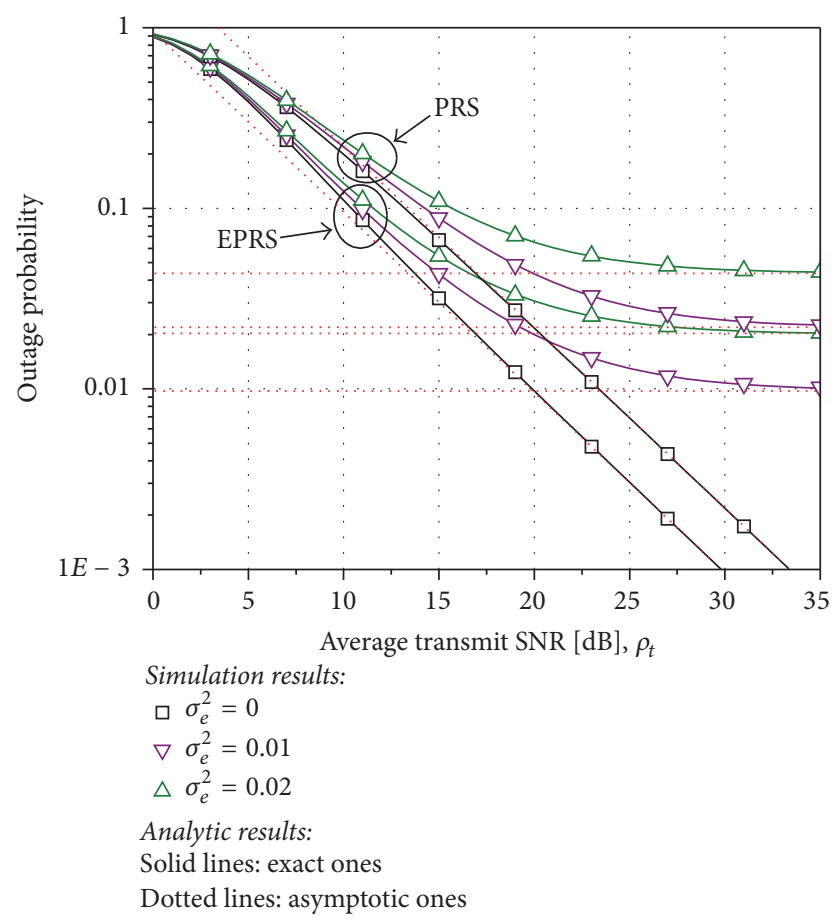

FIGURE 7: Outage probabilities of EPRS and PRS with $\rho_{d}=0.9$ for Case III when $\sigma_{e}^{2}=0,0.01,0.02$.

channel power gap between the first and the second hops increases. Also, such a performance aspect is not affected by $\sigma_{e}^{2}$.

\section{Conclusions}

This paper presents the exact and closed-form expressions for outage probabilities of PRS and EPRS in dual-hop DF relaying systems with channel estimation errors and outdated CSI under nonidentical Rayleigh fading channels. In addition, the expressions for their asymptotic outage probabilities are presented. Numerical results verify the analytic expressions and show that the performance improvement of EPRS over PRS becomes smaller as the feedback delay time increases, but it can be better as the number of relays and the average channel power gap between the first and the second hops increase. Furthermore, the impact of channel estimation errors on the outage performance is much more serious than feedback delay, since the channel estimation errors induce a considerable reduction in the diversity order. Finally, we recognize that a CSI feedback design is much more important for EPRS than PRS, and an advanced channel estimation scheme is necessarily required for both EPRS and PRS in order to maintain the diversity order. Furthermore, when the multiantenna relays are considered, an impact of channel estimation errors and outdated CSI on the system performance may be more serious than the single-antenna scenario. Therefore, further study of the performance analysis of the cooperative relaying system with MIMO configuration is required in the presence of channel estimation errors and outdated CSI.

\section{Competing Interests}

The authors declare that they have no competing interests.

\section{Acknowledgments}

This work was supported by the ICT R\&D program of MSIP/IITP [B0117-16-1005, A Study on Core Technology for Multidimensional Immersive Media Broadcasting and Communication].

\section{References}

[1] J. Sydir, "IEEE 802.16 Broadband Wireless Access Working Group-harmonized contribution on 802.16j (mobile multihop relay) usage models," IEEE 802.16 j Working Group Document, vol. 6 , no. 15, pp. 1-12, 2009.

[2] 3GPP TS 36.216 V10.3.1, Evolved universal terrestrial radio access (E-UTRA); Physical layer for relaying operation, September 2011.

[3] A. Bletsas, A. Khisti, D. P. Reed, and A. Lippman, "A simple cooperative diversity method based on network path selection," IEEE Journal on Selected Areas in Communications, vol. 24, no. 3, pp. 659-672, 2006.

[4] J.-B. Kim and D. Kim, "Exact and closed-form outage probability of opportunistic decode-and-forward relaying with unequalpower interferers," IEEE Transactions on Wireless Communications, vol. 9, no. 12, pp. 3601-3606, 2010.

[5] I. Krikidis, J. Thompson, S. Mclaughlin, and N. Goertz, "Amplify-and-forward with partial relay selection," IEEE Communications Letters, vol. 12, no. 4, pp. 235-237, 2008. 
[6] J.-B. Kim and D. Kim, "Comparison of tightly powerconstrained performances for opportunistic amplify-andforward relaying with partial or full channel information," IEEE Communications Letters, vol. 13, no. 2, pp. 100-102, 2009.

[7] I.-H. Lee, "Outage performance of efficient partial relay selection in amplify-and-forward relaying systems over rayleigh fading channels," IEEE Communications Letters, vol. 16, no. 10, pp. 1644-1647, 2012.

[8] I.-H. Lee and S. Lee, "Bit error probability of decode-andforward relaying with efficient partial relay selection in nonidentical Rayleigh fading channels," IET Communications, vol. 8, no. 15, pp. 2624-2632, 2014.

[9] M. Seyfi, S. Muhaidat, J. Liang, and M. Dianati, "Effect of feedback delay on the performance of cooperative networks with relay selection," IEEE Transactions on Wireless Communications, vol. 10, no. 12, pp. 4161-4171, 2011.

[10] M. Soysa, H. A. Suraweera, C. Tellambura, and H. K. Garg, "Partial and opportunistic relay selection with outdated channel estimates," IEEE Transactions on Communications, vol. 60, no. 3, pp. 840-850, 2012.

[11] D. S. Michalopoulos, H. A. Suraweera, G. K. Karagiannidis, and R. Schober, "Amplify-and-forward relay selection with outdated channel estimates," IEEE Transactions on Communications, vol. 60, no. 5, pp. 1278-1290, 2012.

[12] T. Yoo and A. Goldsmith, "Capacity and power allocation for fading MIMO channels with channel estimation error," Institute of Electrical and Electronics Engineers. Transactions on Information Theory, vol. 52, no. 5, pp. 2203-2214, 2006.

[13] J.-B. Kim, J.-W. Choi, and J. M. Cioff,, "Cooperative distributed beamforming with outdated CSI and channel estimation errors," IEEE Transactions on Communications, vol. 62, no. 12, pp. 4269-4280, 2014.

[14] S. M. Kay, Fundamentals of Statistical Signal Processing: Estimation Theory, Prentice-Hall Press, 1993.

[15] T. R. Ramya and S. Bhashyam, "Using delayed feedback for antenna selection in MIMO systems," IEEE Transactions on Wireless Communications, vol. 8, no. 12, pp. 6059-6067, 2009.

[16] W. C. Jakes, Microwave Mobile Communications, John Wiley \& Sons, 1974.

[17] N. C. Beaulieu and J. Hu, "A closed-form expression for the outage probability of decode-and-forward relaying in dissimilar Rayleigh fading channels," IEEE Communications Letters, vol. 10, no. 12, pp. 813-815, 2006.

[18] J. G. Proakis, Digital Communications, McGraw-Hill, New York, NY, USA, 4th edition, 2001.

[19] 3GPP TR 36.814 V0.4.1, 3rd Generation Partnership Project; Technical specification group radio access network; Further advancements for E-UTRA physical layer aspects (Release 9), February 2009. 


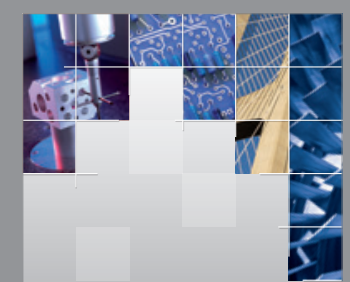

\section{Enfincering}
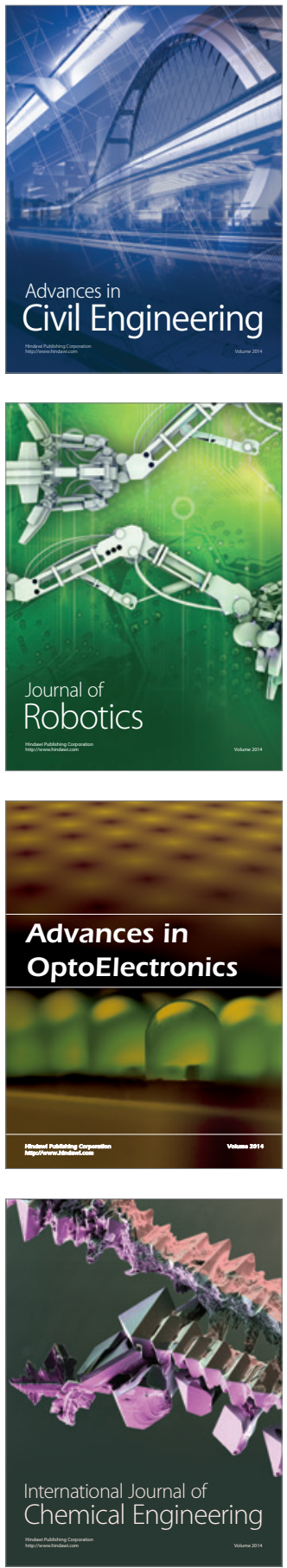

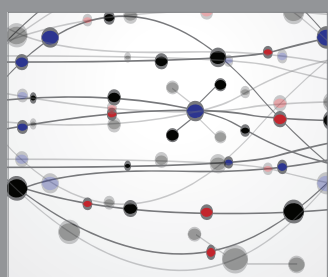

The Scientific World Journal

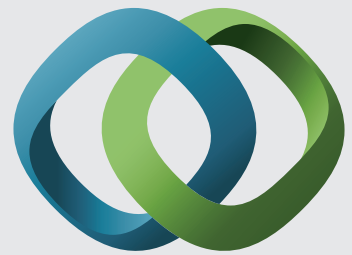

\section{Hindawi}

Submit your manuscripts at

https://www.hindawi.com
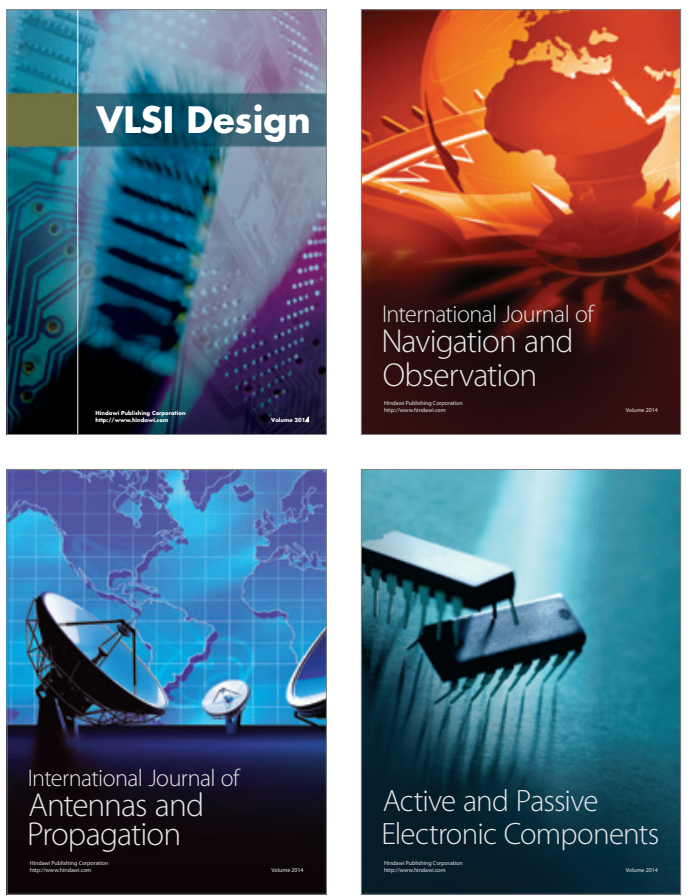
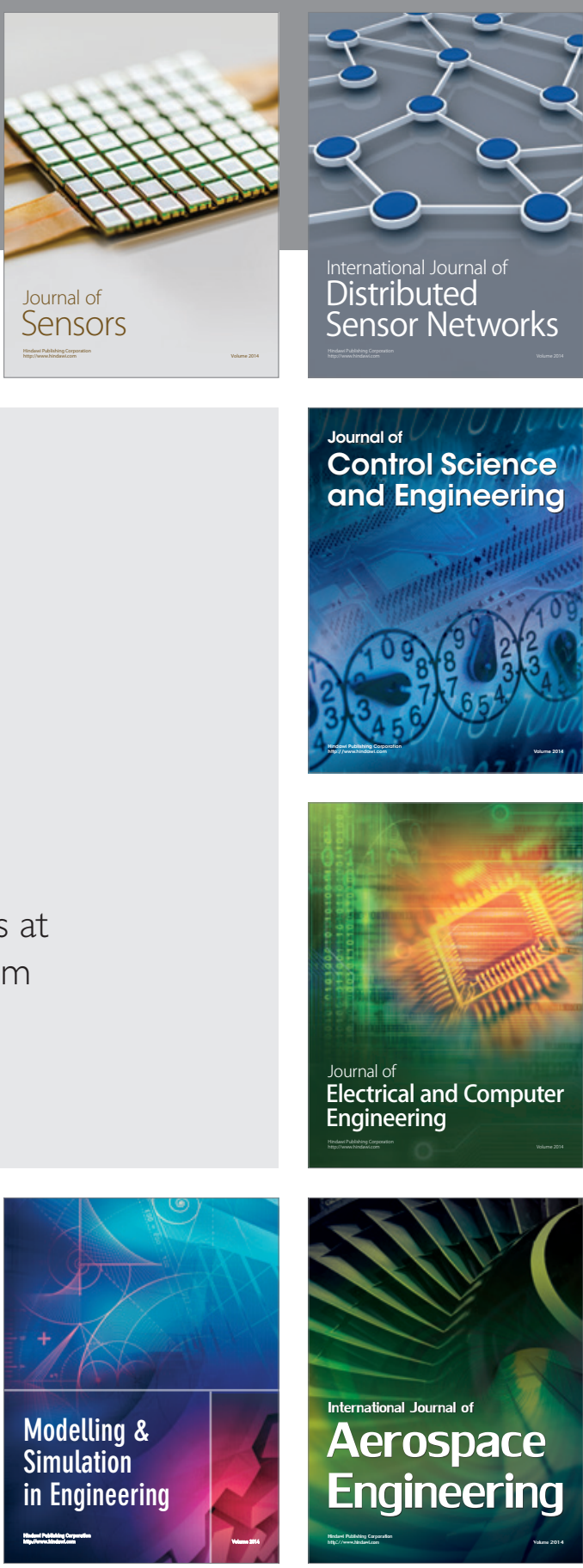

International Journal of

Distributed

Sensor Networks

$-$

Joumal of

Control Science

and Engineering
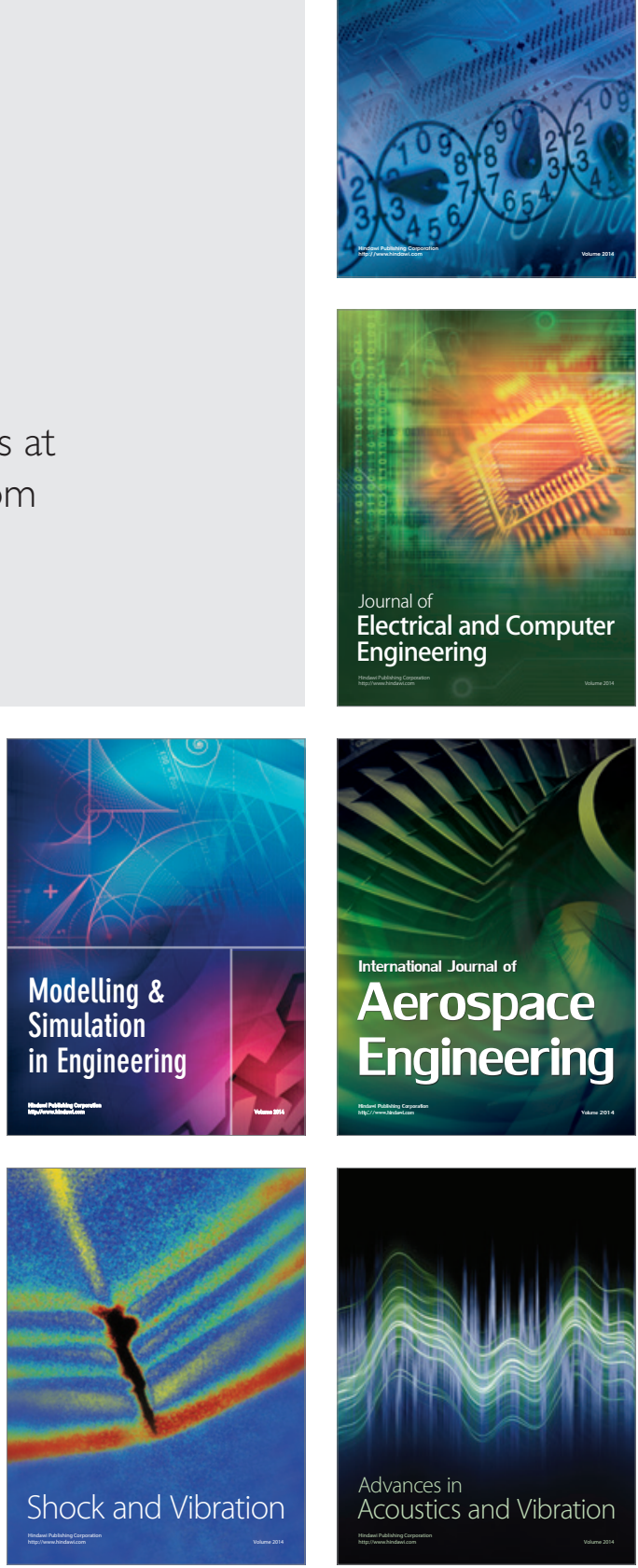